\title{
Temperature as competitive strategy determining factor in pulse-fed aerobic bioreactors
}

\author{
Gerben Roelandt Stouten $\mathbb{1}^{1} \cdot$ Carmen Hogendoorn $^{1,2} \cdot$ Sieze Douwenga $\mathbb{1}^{1,3} \cdot$ Estelle Silvia Kilias ${ }^{4,5}$. \\ Gerard Muyzer $\mathbb{D}^{4} \cdot$ Robbert Kleerebezem ${ }^{1}$
}

Received: 28 August 2018 / Revised: 6 August 2019 / Accepted: 14 August 2019 / Published online: 2 September 2019

(c) The Author(s), under exclusive licence to International Society for Microbial Ecology 2019

\begin{abstract}
Exposing a microbial community to alternating absence and presence of carbon substrate in aerobic conditions is an effective strategy for enrichment of storage polymers (polyhydroxybutyrate, PHB) producing microorganisms. In this work we investigate to which extent intermediate storage polymer production is a temperature independent microbial competition determining factor. Eight parallel bioreactors were operated in the temperature range of $20-40{ }^{\circ} \mathrm{C}$, but intermediate storage polymer production was only obtained at $25-35^{\circ} \mathrm{C}$. Besides PHB production and consumption, cell decay and subsequent cryptic growth on lysis products was found to determine process properties and the microbial community structure at all operational temperatures. At $40^{\circ} \mathrm{C}$ decay processes cannot be overcome with additional energy from storage polymers, and fast-growing microorganisms dominate the system. At $20^{\circ} \mathrm{C}$, highly competitive communities with ambiguous storage properties were enriched. The results described here demonstrate that a rigorous experimental approach could aid in the understanding of competitive strategies in microbial communities.
\end{abstract}

\section{Introduction}

Natural microbial communities are complex biological matrices, which typically contain thousands of different types of microorganisms [1]. Understanding the ecological and evolutionary implications of this diversity is one of the

Supplementary information The online version of this article (https:// doi.org/10.1038/s41396-019-0495-8) contains supplementary material, which is available to authorized users.

$\square$ Gerben Roelandt Stouten

G.R.Stouten@tudelft.nl

1 Environmental Biotechnology, Department of Biotechnology, Delft University of Technology, Delft, The Netherlands

2 Department of Microbiology, IWWR, Radboud University Nijmegen, Nijmegen, The Netherlands

3 Systems Bioinformatics, AIMMS, VU University Amsterdam, Amsterdam, The Netherlands

4 Microbial Systems Ecology, Department of Freshwater and Marine Ecology, Institute for Biodiversity and Ecosystem Dynamics, University of Amsterdam, Amsterdam, The Netherlands

5 Biosciences, University of Exeter, Exeter, UK central topics in Biology [2]. With the arrival of Next Generation Sequencing techniques, a wealth of genomic information is now available on the composition of microbial communities in both natural and man-made ecosystems. While the genotypical makeup of microbial communities can be well described, our knowledge of phenotypical aspects like biochemical functionality and interspecies dependencies is still wanting [3-6]. The changes in the microbial community composition are a key research topic in fields like environmental biotechnology, gut microbiology, and agricultural microbiology [7-9]. By using well-controlled bioreactors, the development of microbial community structure and functional properties can be investigated with more accurateness then by studying natural habitats. A profound limitation remains in the resolution of functional characterization, mostly due to the required sampling and analysis effort and its possible interference with bioreactor operation. Therefore, infrequent or limited description of the functional development often restricts the linking of community structure dynamics to changes in functionality, and is thereby a bottleneck in our understanding of microbial community development and succession in ecosystems upon exposure to different environmental conditions. 
The ever-present microbial diversity is of specific importance in environmental biotechnological processes, such as resource recovery facilities, that rely on selective conditions to maintain stable process operations. Microbial ecology research in many cases aims for identifying the mechanisms driving changes in functionality $[10,11]$. Functional changes are eventually reflected in the microbial community structure that establishes. An increased understanding of the relationship between the functional performance and microbial community structure will lead to improved microbial ecosystem understanding and enables improved process design and operation, and facilitate the development of novel microbial community-based processes. A significant contribution to this understanding will come from observation-based research where a single process condition is changed in a selective environment. By operating multiple parallel systems, microbial community related questions regarding inoculum, medium composition, reproducibility, and bioreactor operation can be addressed properly. While attempts to combine datasets from different studies to obtain a comprehensive understanding of the influence of process parameters on process performance are hampered by the fact that almost all studies are performed in a dissimilar way. The limitations in our understanding of microbial competition and coexistence should therefore benefit from more rigorous efforts to compare the structure and function development of a microbial community at specific selective conditions.

To enable this research intensification, a high-resolution system characterization platform has been developed. In a separate manuscript (in preparation), system characterization by high-resolution online data consolidation in dynamic systems is further expanded upon [12]. The study presented here shows an implementation of research intensification to investigate the influence of temperature on pulse-fed enrichments of bacteria in sequencing batch reactors (SBR). The experimental setup of this study is analogous to previous studies [13-16], but now encompasses a temperature range from 20 to $40{ }^{\circ} \mathrm{C}$ in eight bioreactors. As is well documented in those studies, pulse-fed SBR, operated with a single nutrient limitation establish a feast-famine enrichment. Microorganisms can compete for the limiting nutrient with at least two distinct ecological strategies. 'Growers' directly produce catalytic biomass from the substrate supplied, while 'hoarders' store the substrate as internal storage compounds and metabolize them later to catalytic biomass. The relative biochemical simplicity of storage polymer production compared with catalytic biomass production as well as the lower energy requirements have been proposed to provide a kinetic competitive advantage to hoarders compared with fastgrowing microorganisms. This possibility of feast-famine systems to enrich for microorganisms that produce storage polymers has sizeable industrial potential, especially in the field of resource recovery from waste streams [17]. The type of storage polymer that is formed depends on the available substrate. Waste streams are generally rich in volatile fatty acids, which are consumed to produce polyhydroxyalkanoates (PHA), which can serve as a renewable resource for the production of bioplastics and biochemicals [18]. Polyhydroxybutyrate (PHB) is the most common type of PHA that is produced by a variety of bacteria.

This work aims to identify the role of storage polymers in microbial competition at different operational temperatures. The use of parallel-operated reactors combined with online data collection with community structure analysis, this study furthermore strives to align the structural and functional development in time of the microbial community as a function of temperature. The methodology used in this work provides insight in microbial community structure development and contributes to future advancements in microbial community-based bioprocess development, of which PHB production by mixed microbial cultures is a prime example. This paper focuses on the implementation of high-resolution system characterization, and specifically on the comparative results that can be discussed due to the parallel nature of this work.

\section{Methods}

\section{Sequencing batch reactors}

Eight bioreactors (glass, double-jacket, $1.4 \mathrm{~L}$ working volume) (Applikon, the Netherlands) were operated in parallel, under nonsterile conditions for 208 cycles, following the operating strategy as outlined in Supplementary Table 1. Stirring was set to $700 \mathrm{rpm}$ (SC4, DASGIP, Germany), air gas flow was controlled by mass flow controllers (MX44, DASGIP, Germany) at $400 \pm 2 \mathrm{mln} / \mathrm{min}$. Reactors were temperature controlled by means of a water jacket and thermostat bath (Lauda, Germany) at different operational temperatures $\left(20^{\mathrm{A}, \mathrm{B}}, 25,30^{\mathrm{A}, \mathrm{B}}, 35\right.$ and $40^{\mathrm{A}, \mathrm{B}} \pm$ $0.5^{\circ} \mathrm{C}$, duplicates are indicated with a superscript), $\mathrm{pH}$ was maintained at $7.0 \pm 0.1$ using $1 \mathrm{M} \mathrm{HCl}$ and $0.5 \mathrm{M} \mathrm{NaOH}$. The 48 pumps for feeding, effluent removal and $\mathrm{pH}$ control were controlled by a hardware abstraction layer (HAL, TU Delft, Netherlands). The HAL in turn was controlled by a PC using custom scheduling software (D2I, TU Delft, Netherlands). D2I was also used for data acquisition of the online measurements, i.e., dissolved oxygen (DO), $\mathrm{pH}$, acid/base dosage, feed balance, in-gas/off-gas composition.

The removal of $50 \%$ of the reactor volume at the end of each cycle resulted in a solid and hydraulic retention time of 1 day (SRT and HRT, respectively). The reactors were cleaned twice per week to reduce the influence of wall 
growth. The reactors were inoculated at the same time with a mixture (Supplementary Fig. S12) of aerobic activated sludge from the municipal wastewater treatment plant Harnaschpolder in Delft, the Netherlands, (operational temperature $10-20^{\circ} \mathrm{C}$ ), and process water from a papermill from Hoogezand, the Netherlands, (operated at $35-45^{\circ} \mathrm{C}$ ). The medium was prepared on molality (moles $/ \mathrm{kg}[\mathrm{m}]$ ) as dosing is performed through a balance. Each cycle a total of $680 \mathrm{~g}$ nutrients were dosed: $20 \mathrm{~g}$ acetate feed stock $(1 \mathrm{~m})$,

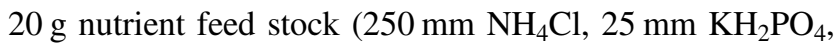
$6.25 \mathrm{~mm} \mathrm{MgSO}_{4} .7 \mathrm{H}_{2} \mathrm{O}, 7.5 \mathrm{~mm} \mathrm{KCl}$, and $50 \mathrm{~g} / \mathrm{L}$ trace element solution [19]), and $640 \mathrm{~g}$ distilled water. With a working volume of $1.4 \mathrm{~L}$ the peak concentration of acetate at the start of each cycle is $28.5 \mathrm{Cmmol} / \mathrm{L}$. Throughout the cycle, $\mathrm{pH}$ correction results in the addition of $\sim 20 \mathrm{~g}$ of $1 \mathrm{M}$ $\mathrm{HCl}$ to the bioreactor.

\section{Cycle and fed-batch measurements}

Cycle measurements of normal SBR operations were performed to fully characterize the bioreactor and verify online measurements. Measurements are performed as described by Johnson [13]. After 146 enrichment cycles the bioreactors were exposed to a fed-batch experiment in order to determine the maximum PHB production capacity of the biomass. After collecting the effluent of cycle 145 and storing it at $4{ }^{\circ} \mathrm{C}$, a fed-batch experiment was performed by adding $660 \mathrm{~g}$ acetate and ammonium-free medium to the remaining biomass. After $1 \mathrm{~h}$, a $28.5 \mathrm{Cmmol} / \mathrm{L}$ sodium acetate pulse was given. For pH control, $1 \mathrm{M}$ acetic acid and $0.5 \mathrm{M} \mathrm{NaOH}$ were used, thereby constructing a feed on demand strategy. Fed-batch experiments ran for $12 \mathrm{~h}$, after which the total reactor content was replaced by the stored biomass.

\section{Temperature disturbance}

From cycle 150 till cycle 200 several temperature disturbance experiments were performed with the enriched biomass. The bioreactors continued their normal SBR operations, with the exception that the bioreactor temperature was increased or decreased by $5^{\circ} \mathrm{C}$ after extracting the effluent. Within $1 \mathrm{~h}$, the desired temperature in the bioreactor was reached and the 28.5 Cmmol/L acetate pulse was given. The disturbance ran for two cycles, after which the temperature was restored to normal. The reactors were allowed to stabilize for ten cycles before another disturbance experiment was performed.

\section{Analytical methods}

Online measurements consisted of pH, DO (Mettler Toledo, USA) as a percentage of air saturation, acid and base dosage through integrated revolution counter (MP8, DASGIP, Germany), feed balance (LE34001S, Sartorius, Germany), and high frequency in-gas and off-gas composition analysis through mass spectrometry (PRIMA BT Benchtop, Thermo Scientific, UK). Offline measurements were performed for acetate, ammonia, PHB, total suspended solids, and volatile suspended solids (VSS) as described by Johnson [13]. Catalytic biomass is determined by subtracting PHB from VSS measurement. Catalytic biomass is assumed to have a composition of $\mathrm{CH}_{1.8} \mathrm{O}_{0.5} \mathrm{~N}_{0.2}$ [20].

\section{Functionality characterization from online data}

Identification of functional changes is performed by observing dynamics in key indicators, which are calculated for each 12-h cycle. Indicators were derived from principle system characterization of online measurements. Online measurements can, under well-defined conditions, be used for a full system characterization (manuscript in preparation) [12].

An important indicator in feast-famine systems is the end of the feast phase, where external substrate is depleted. The feast length is indicative of the substrate uptake rate and allows for further characterization of the behavior of the biomass in the feast and famine phase. The feast length is derived from the inflection point of the oxygen transfer rate (OTR), which is a strong indicator for the depletion of extracellular substrate [21].

The here proposed key functionality indicators are specific to the aerobic feast-famine system. Both indicators are normalized values, making it possible to compare bioreactors with different process parameters. $\mathrm{O}_{2 \text {,feast }} \%$ : the fraction of oxygen consumed in the feast phase $\left(\mathrm{O}_{2 \text {,feast }}\right.$ $\left.\left(\mathrm{O}_{2 \text {,feast }}+\mathrm{O}_{2 \text {,famine }}\right)\right)$. OTR $\mathrm{O}_{\text {ratio }}$ : the ratio of the initial to the maximum OTR in the feast phase $\left(\mathrm{OTR}_{\text {start-feast }} / \mathrm{OTR}_{\max }\right)$. The sharp transition of the feast to the famine phase suggests that the biomass has a high substrate affinity as expressed by a low $K_{\mathrm{m}}$ value. How $\mathrm{O}_{2 \text {,feast }} \%$ and $\mathrm{OTR}_{\text {ratio }}$ are related to different functionalities is illustrated in Fig. 1. Shifts in functionality are identified by observing changes throughout the enrichments of these characteristic indicators.

\section{Parameter identification}

Cycle experiments were used to identify biomass specific parameters according to the approach proposed by Johnson [21]. After sampling and physicochemical corrections, the carbon and electron balances closed for $95 \%( \pm 5)$. The corrected data was evaluated in a metabolic model to determine the biomass specific reaction rates and other kinetic parameters, as described by Marang [22]. A biomass viability parameter $\left(\mathrm{k}_{\mathrm{d}}\left[\mathrm{h}^{-1}\right]\right)$ was added to the model to allow modeling of biomass decay [23]. Weighing factors in the squared errors were made proportional to measurement errors [24]. Adaptations to the model of Johnson can be found in Supplementary Materials. 


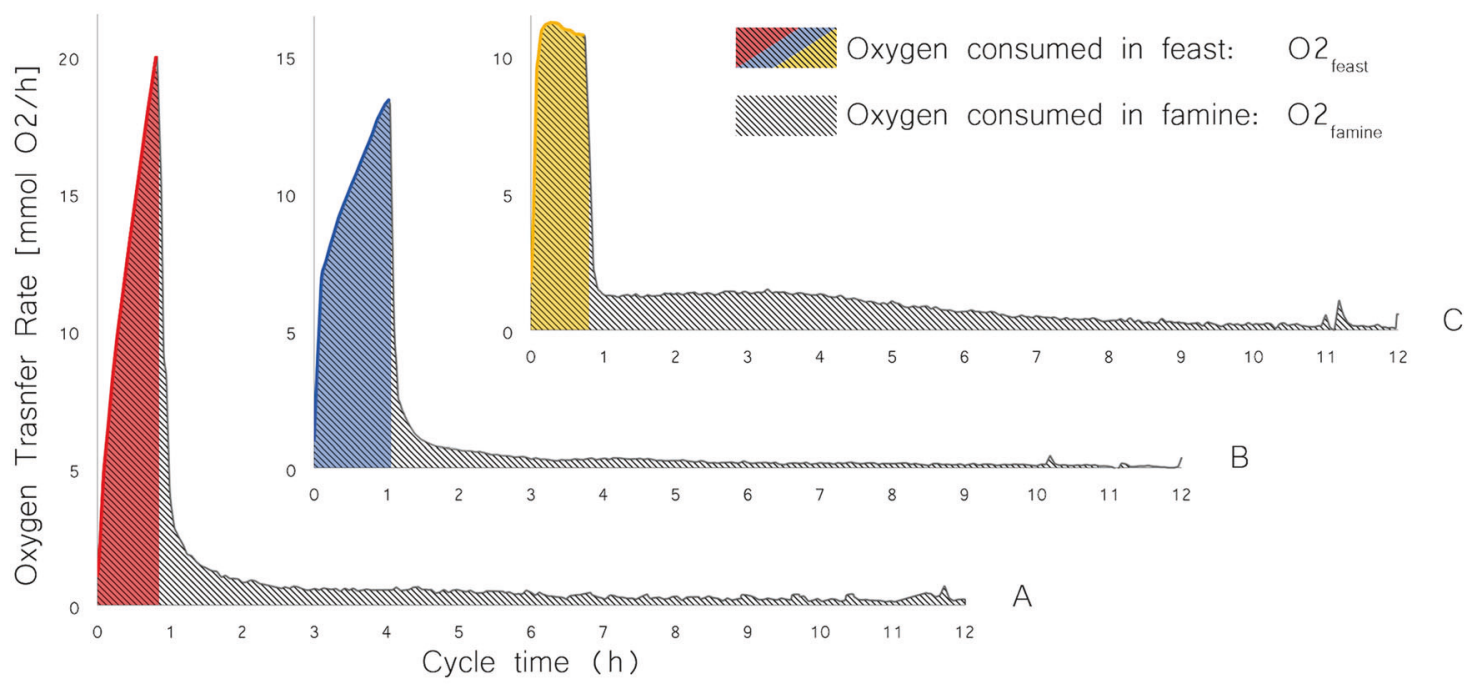

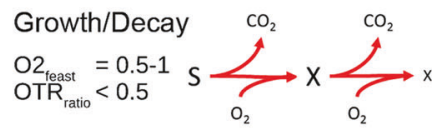

Growth
$\mathrm{O}_{\text {feast }}=1$
$\mathrm{OTR}_{\text {ratio }}=0.5$

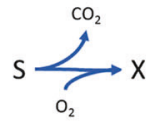

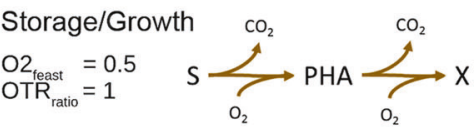

Fig. 1 Overview of three distinct functional responses of different microbial communities to a new feed pulse at $t=0$ after half of the biomass is removed. The colored regions indicate the feast period in which external substrate is available, all systems have a comparable feast length of $1 \mathrm{~h}$, but different functional responses. Response A (red) shows a significant increase in respiration activity, indicating that either inactive biomass takes time to reactivate, or a relatively large fraction of biomass has decayed and needs to be replenished. Response B (blue) shows a doubling in respiration activity, which is associated with high cell viability and production of new catalytic biomass.
Response C (yellow) shows a constant respiration activity, indicating that no new catalytic biomass is produced in the feast phase. The normalized change in oxygen transfer rate (OTR) throughout the feast

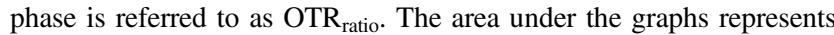
the total oxygen consumption, which can be divided in oxygen con-

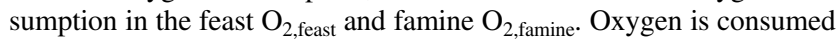
in the famine phase for growth on storage polymers and cell maintenance. Substrate $(\mathrm{S})$ is either directly converted to biomass $(\mathrm{X})$ or first to $\mathrm{PHB}$, and consequently to biomass

\section{Community structure analysis by $16 \mathrm{~S}$ rRNA gene amplicon sequencing}

Microbial community composition analysis was performed by taking a $15 \mathrm{ml}$ sample from the bioreactor at the end of a cycle and centrifuging it for $10 \mathrm{~min}$ at $5000 \mathrm{~g}$. The pellet was transferred to a $2 \mathrm{ml}$ Eppendorf tube and stored at $-80^{\circ} \mathrm{C}$, awaiting further treatment. Genomic DNA of 146 samples (18 time points for eight bioreactors + two replicates of the inoculum) was extracted with the PowerSoil DNA Isolation Kit (Mo Bio Laboratories, Inc., Carlsbad, CA, USA) according to the protocol as described by the manufacturer. The extracted DNA was quantified using absorption spectrophotometry at $280 \mathrm{~nm}$. 16S rRNA gene amplicon sequencing was performed on an Illumina Miseq sequencer by the company MR DNA. The sequences have been stored in GenBank under project number PRJNA448046.

\section{Focus of sequencing data for this study}

This study aims at comparing the developments in microbial community structure and functional behavior of the reactors upon exposure to a selective environment. To that end the $16 \mathrm{~S}$ rRNA gene amplicon sequencing data is processed by the following QIIME pipeline.

The merged $16 \mathrm{~S}$ Illumina reads file (.fastq) together with the mapping file (.txt) were processed and sorted using a phred threshold of 29 and read length between 398 and 450 base pairs on QIIME version 1.9.1 (split_libraries_fastq.py) [25]. Chimera identification was performed with USEARCH [26] and the RDP reference database [27]. Quality filtered reads were clustered to OTUs at $97 \%$ similarity (pick_otus. py) [28], singletons were dropped. Sequence alignment is performed with the MUSCLE [29] aligner, and taxonomy was assigned with the RDP classifier with a confidence of 0.9 .

Brooks [30] and Sinha [31] showed that biases in $16 \mathrm{~S}$ studies due to sample handling, DNA extraction and PCR amplification strongly restricts the quantitative interpretation of sequencing results. The processing steps affect different bacteria in different ways, resulting in amplified and suppressed observed proportions of a community. The high technical reproducibility does allow visualization of relative community dynamics in time throughout enrichments. Plotting the natural logarithm of the relative abundance of 

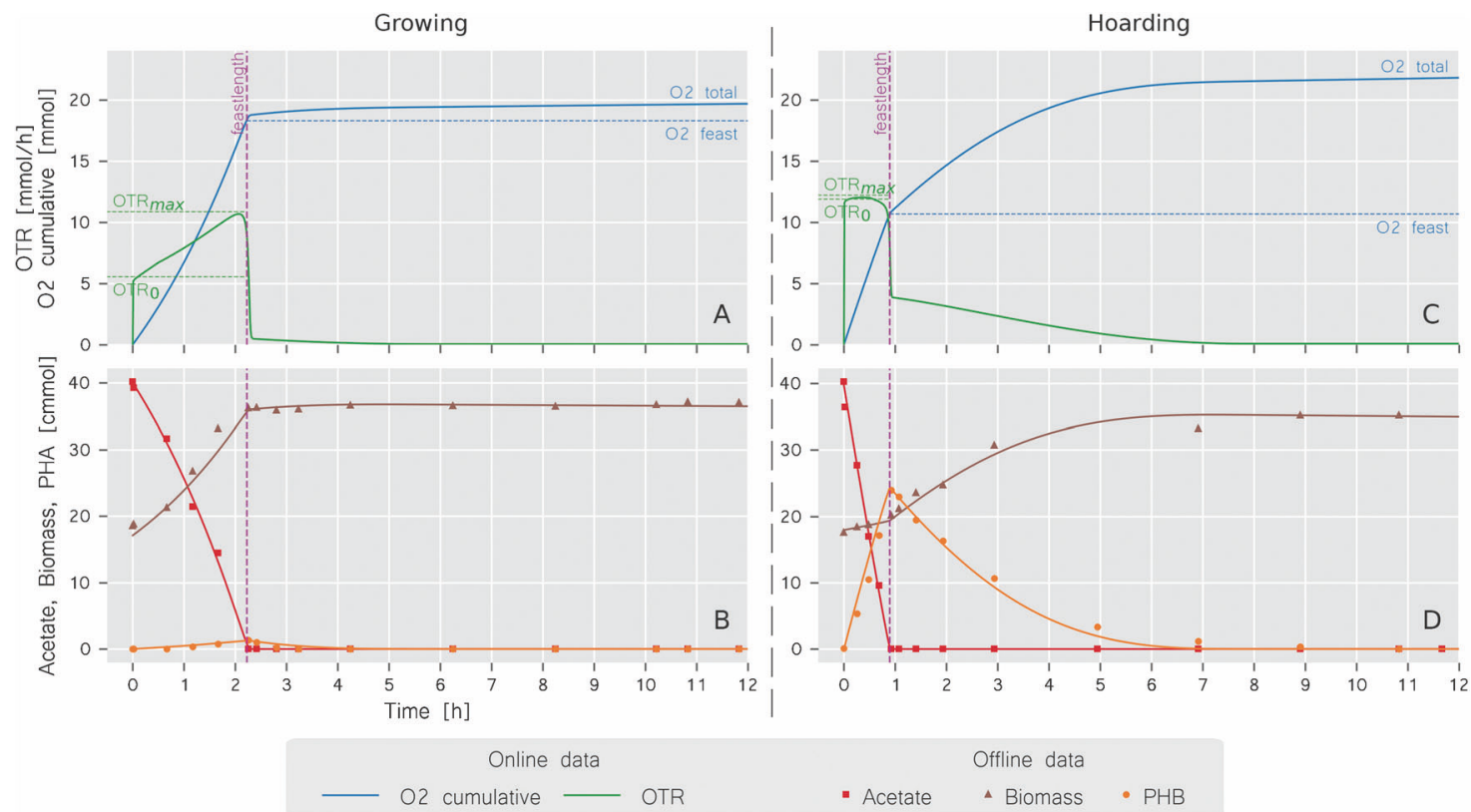

Fig. 2 Online and offline data of R20A (a, b) and R30A (c, d). System characterizations by online measurements are based on key indicators, represented by text labels and dashed lines in the online data (a, c): oxygen transfer rate (OTR) and oxygen consumption in the feast and famine $\left(\mathrm{O}_{2}\right)$. These characterizations align with offline $(\mathbf{b}, \mathbf{d})$ measurements. Clear differences are observed between growing

species allows for a clearer interpretation of community shifts. The sequencing results yielded between 48.000 and 117.000 paired-end reads per sample. Community dynamics that occur below 1000 reads cannot be represented in a classical stacked bar plot as these dynamics occur within $1 \%$ of the relative abundance. Logarithmic representations utilize the whole measurement range of the $16 \mathrm{~S}$ sample reads, leading to deeper visualizations of community dynamics, and thereby capture the gradual dynamics that can occur in communities (Supplementary Fig. S11).

Correlations between community composition and functionality were performed using Pearson's method (Supplementary Table 2). The relative abundance of each OTU for each bioreactor is correlated against the feast length, $\mathrm{O}_{2 \text {,feast }} \%$, and $\mathrm{OTR}_{\text {ratio }}$ for that bioreactor at the 18 sampling time points.

\section{Results}

\section{Characterization of microbial community function from online data}

Eight bioreactors, pulse-fed with acetate and operated under aerobic conditions for 100 days, were seeded with activated sludge and operated identically, except for the operational temperature. Bioreactors were operated at 20, 25, 30, 35, and bioreactors (R20A-a, b) and hoarding bioreactors (R30A-b, d) allowing for online characterization of functional behavior without offline measurements. Solid lines in offline data represent the fitted model based on [21, 22], where ammonium measurements are used to determine the biomass production

$40{ }^{\circ} \mathrm{C}$. Duplicate reactors were operated at 20,30 , and $40{ }^{\circ} \mathrm{C}$. The development of the functional properties of the microbial communities in the bioreactors was determined by online measurements during the enrichments. Online measured variables were $\mathrm{O}_{2}$ and $\mathrm{CO}_{2}$-off gas, base and acid dose, bioreactor temperature, $\mathrm{DO}$ and $\mathrm{pH} . \mathrm{O}_{2}$-off gas measurements were used to functionally characterize the process, whereas DO measurements were used to verify $\mathrm{O}_{2}$-off gas data. $\mathrm{CO}_{2}$-off gas measurements were used for solving the carbon balance over each operational cycle, where acid and base dose were used for identifying the stoichiometric factor for protons in the metabolic stoichiometry.

Two variables calculated from $\mathrm{O}_{2}$-off gas data were found to correlate well with offline functionality characterizations: the fraction of oxygen consumed in the feast phase $\left(\mathrm{O}_{2 \text {,feast }} \%\right)$, and the normalized change in OTR throughout the feast phase, expressed as the ratio of maximum and initial OTR $\left(\mathrm{OTR}_{\text {ratio }}\right)$. These process variables correlate with the growth and hoarding strategies as follows:

The growing strategy is characterized by the production of catalytic biomass during the feast phase, and relatively little respiratory activity in the famine phase. The biomass in bioreactor A operated at $20^{\circ} \mathrm{C}(\mathrm{R} 20 \mathrm{~A})$ exhibited a characteristic growing strategy as shown in Fig. 2. The growth strategy was identified by online measurements (Fig. 2a) and confirmed by offline cycle measurements (Fig. 2b). In line with the growth strategy, oxygen uptake in the cycle shown in 
Fig. $2 \mathrm{~b}$ occurs mainly in the feast phase, and the $\mathrm{O}_{2 \text {,feast }} \%$ is high (87\%). Minor amounts of respiration occurring in the famine phase can be attributed to completion of the growth cycle, maintenance, and/or endogenous respiration. The growth strategy is confirmed by the increase in respiration rate during the feast phase. In absence of storage compounds, the doubling of the biomass during the feast phase as imposed by the exchange ratio of $50 \%$ corresponds to an $\mathrm{OTR}_{\text {ratio }}$ of 0.5 , as is observed for R20A and shown in Fig. 2a.

The hoarding strategy is characterized by the production of PHB during the feast phase, and growth on PHB throughout the famine phase. The biomass in bioreactor $\mathrm{A}$ operated at $30{ }^{\circ} \mathrm{C}$ (R30A) developed into a characteristic hoarding strategy within 30 days of operation as shown in Fig. 2. This hoarding strategy can directly be observed from online measurements (Fig. 2c) and was confirmed by offline cycle measurements (Fig. 2d). Respiration in the famine phase is dominated by the oxidation of PHB, coupled to biomass production as confirmed by offline measurement of PHB degradation and biomass production. Carbon and electron balances close within $95 \%$. The cycle of R30A shown in Fig. 2c has an $\mathrm{O}_{2 \text {,feast }} \%$ of $50 \%$, signifying that more than $95 \%$ of the catalytic biomass is produced in the famine phase (see Supplementary Material for details on the calculation). Furthermore, the absence of growth during the feast phase is confirmed by the constant OTR in the feast phase as reflected by an OTR $_{\text {ratio }}$ of 1.0.

The online determined feast length, $\mathrm{OTR}_{\text {ratio }}$, and the $\mathrm{O}_{2}$, feast $\%$ were found to correlate well with the functionalities identified from offline cycle measurements as shown in Fig. 2. It was concluded that online characterization could effectively be used as identifier of the dominant competitive strategy as well as the process development and stability in time.

\section{Comparing structural and functional development over time}

Eight reactors were operated for 208 cycles. For brevity, only a detailed overview of the development of the feast length, $\mathrm{O}_{2 \text {,feast }} \%$, $\mathrm{OTR}_{\text {ratio }}$, and microbial community structure, for bioreactor R30A is presented here (Fig. 3). The full datasets for the other seven bioreactors are available in Supplementary Figs. S1-S8.

The competition for the limiting substrate is reflected by a downward trend of the feast length throughout the enrichment (Fig. 3a). The reduction of the feast length in time does not elude which competitive strategy dominates or when a functionality shift occurs. The plot of the $\mathrm{O}_{2 \text {,feast }}$ $\%$ shows a clear decrease from 80 to $50 \%$ from cycle 50-65 approximately (Fig. 3b). The plot of the OTR ratio $_{\text {shows a }}$ shift towards a value of 1 in the same period (Fig. 3c). This demonstrates a clear shift from the growth to hoarding competitive strategy, as confirmed by the PHB fraction measured at the end of the feast phase (green squares in Fig. 3c representing $\left.g_{\mathrm{PHB}} / g_{\mathrm{VSS}}\right)$.

Eighteen biomass samples were collected from each bioreactor for microbial community composition analysis. The relative abundance of eight OTUs is displayed in Fig. 3d. The bar plot representation allows for identification of community dynamics and stability of relatively abundant OTUs. The natural logarithm plot of the relative abundance (Fig. 3e) gives a clearer interpretation of time-dependent community shifts by visualizing the whole measurement range of $16 \mathrm{~S}$ amplicon sequencing (see 'Methods' section).

Comparison of the $\mathrm{O}_{2 \text {,feast }} \%$ and $\mathrm{OTR}_{\text {ratio }}$ data with the natural relative abundance plot shows that the upcoming of Plasticicumulans acidivorans in bioreactor R30A is correlated with a shift in functionality from growth to hoarding (Pearson $r=0.920, p \ll 0.001, n=18$, see Supplementary Table 2). The culture that established within the first 50 cycles was starting to be washed out from the moment that $P$. acidivorans became dominant. The rate of exponential washing-out or growing-in results in trends in Fig. 3e. The slopes reflect the difference in substrate uptake rate compared with its competitors. P. acidivorans increased its relative abundance approximately with $30 \%$ each cycle, during its in-growing period. Acinetobacter sp. slowly washed out at less than 5\% every cycle (see Supplementary Materials).

A family of microorganisms that is not commonly associated with fast growth or the production of storage polymers, but is abundantly present in all bioreactors, is the Flavobacteriaceae. This family is well known for the degradative abilities of macromolecules such as proteins and polysaccharides [32]. Most other relatively abundant bacteria are associated with acetate metabolism, e.g.,: Acinetobacter [33], Comamonas [34], Sphingomonas [35], Plasticicumulans [36], and Zoogloea [37]. A literaturesupported overview of the functional capabilities of abundant genera is included in the Supplementary Materials. This information is indicative of the roles that microbes can play in the rather restrictive environment.

\section{Categorizing enrichments based on displayed functionality}

The kinetic performance of the enriched biomass in all eight systems was evaluated in detail by cycle measurements at the end of the enrichment when a functionally steady process was established. An overview of the observed and model-derived parameters is given in Table 1 . The eight bioreactors can be divided in three groups, based on the 


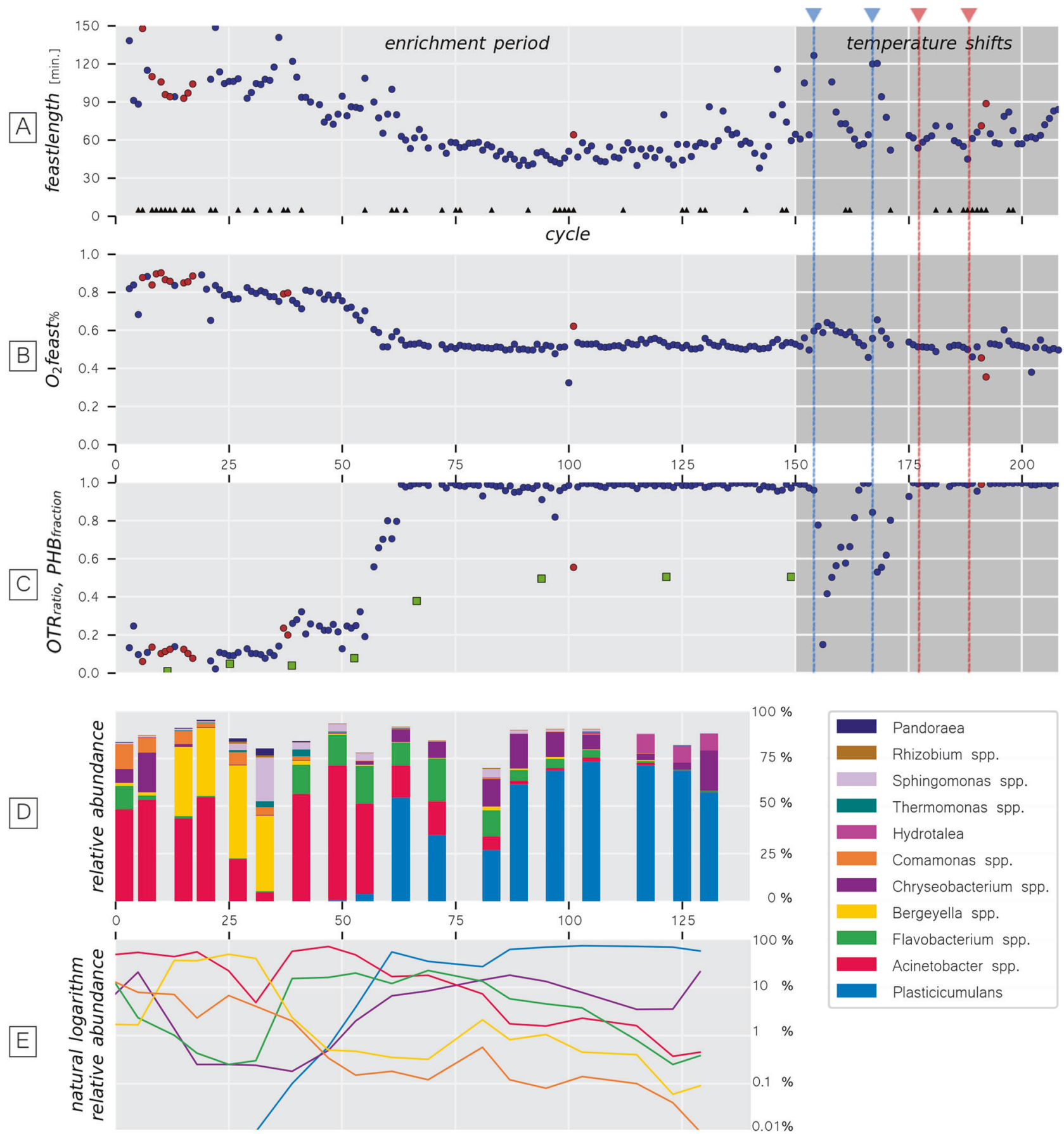

Fig. 3208 enrichment cycles of R30A: overview of characteristic values (a: feast length, b: $\mathrm{O}_{2 \text {,feast }} \%$, c: $\mathrm{OTR}_{\text {ratio }}$ and $\mathrm{PHB}_{\text {fraction }}$ ) and community structure (d: relative abundance, e: natural logarithm of relative abundance of selected species). Upward triangles in subfigure a indicate cycles where the reactor was cleaned. Red circles indicate cycles where reactor operation was disturbed by excessive foaming, $\mathrm{pH}$ disturbance or other manual interventions. The grayed-out region indicates the time period in which temperature shift experiments were

functionality displayed at the end of the enrichment: Growers: R20A, R40A, R40B; Hoarders: R25, R30A, R30B, R35; and Mixed: R20B. The results of representative performed. Dashed vertical lines indicate specific temperature disturbance experiments (blue: temperature decrease, red: temperature increase). In subfigure c, the intermittently measured PHB fraction $\left(g_{\mathrm{PHB}} / g_{\mathrm{VSS}}\right)$ at the end of the feast phase is plotted as solid green squares. The relative abundance plot shows the eleven most abundant species that makeup over $90 \%$ of all reads, other species are not plotted and are reflected as the missing fractions in plot $\mathrm{D}$

feast-famine cycles for growers and hoarders are shown in Fig. 2. For full experimental data on all bioreactors the reader is referred to the Supplementary Figs. S1-S8. 
Table 1 Overview of observed variables, model-derived yields and biomass specific rates, and characteristic values derived from online measurements during normal SBR operation (averages and standard deviation of ten consecutive cycles). Dominant microorganisms based on $16 \mathrm{~S}$ gene sequencing results. PHB content results from normal cycle operation $\left(\mathrm{PHB}_{\text {end-feast }}\right)$ and accumulation experiments $\left(\mathrm{PHB}_{\max }\right)$

\begin{tabular}{|c|c|c|c|c|c|c|c|c|c|}
\hline Bioreactor & & $\mathrm{R} 20 \mathrm{~A}$ & $\mathrm{R} 20 \mathrm{~B}$ & $\mathrm{R} 25$ & $\mathrm{R} 30 \mathrm{~A}$ & R30B & $\mathrm{R} 35$ & $\mathrm{R} 40 \mathrm{~A}$ & $\mathrm{R} 40 \mathrm{~B}$ \\
\hline \multicolumn{10}{|l|}{ SBR cycles } \\
\hline \multicolumn{10}{|l|}{ Observed } \\
\hline Feast length & {$[\mathrm{min}]$} & $140 \pm 3$ & $143 \pm 8$ & $77 \pm 4$ & $50 \pm 4$ & $54 \pm 4$ & $46 \pm 3$ & $53 \pm 1$ & $65 \pm 4$ \\
\hline $\mathrm{PHB}_{\text {end-feast }}$ & [wt\%] & $4 \pm 2$ & $30 \pm 3$ & $51 \pm 1$ & $52 \pm 1$ & $51 \pm 1$ & $48 \pm 1$ & $0 \pm 0$ & $0 \pm 0$ \\
\hline \multicolumn{10}{|l|}{ Model } \\
\hline$q_{\mathrm{s}, \max }$ & {$[\mathrm{Cmol} / \mathrm{Cmol} \mathrm{h}]$} & 0.7 & 0.8 & 1.9 & 2.3 & 2.3 & 2.8 & 3.5 & 1.9 \\
\hline$q_{\mathrm{phb}, \max }$ & {$[\mathrm{Cmol} / \mathrm{Cmol} \mathrm{h}]$} & 0.0 & 0.2 & 1.1 & 1.6 & 1.5 & 1.7 & 0.0 & 0.0 \\
\hline$\mu_{\text {feast }}$ & {$[\mathrm{Cmol} / \mathrm{Cmol} \mathrm{h}]$} & 0.37 & 0.16 & 0.07 & 0.08 & 0.09 & 0.14 & 1.54 & 0.65 \\
\hline$Y_{\mathrm{sx}}$ & {$[\mathrm{Cmol} / \mathrm{Cmol}]$} & 0.51 & 0.47 & 0.50 & 0.48 & 0.47 & 0.45 & 0.41 & 0.40 \\
\hline$k_{\mathrm{d}}$ & {$[\mathrm{Cmol} / \mathrm{Cmol} \mathrm{h}]$} & 0.01 & 0.00 & 0.00 & 0.01 & 0.01 & 0.02 & 0.06 & 0.02 \\
\hline \multicolumn{10}{|l|}{ Characterization } \\
\hline Group $_{\text {functionality }}$ & & Grow & Mixed & Hoard & Hoard & Hoard & Hoard & Grow & Grow \\
\hline OTR $_{\text {ratio }}$ & {$[-]$} & 0.52 & 0.71 & 1.0 & 1.0 & 1.0 & 0.95 & 0.19 & 0.35 \\
\hline \multicolumn{9}{|l|}{ dominant m.o. ${ }^{\mathrm{a}}$} & 76 \\
\hline $\begin{array}{l}\text { Growing } \\
\text { Hoarding }\end{array}$ & & a.spp. & $\begin{array}{l}\text { a.spp. } \\
\text { z.sp. }\end{array}$ & p.a. & p.a. & p.a. & $\begin{array}{l}\text { a.spp. } \\
2 s p .{ }^{\mathrm{b}}\end{array}$ & a.spp. & a.spp. \\
\hline $\begin{array}{l}\text { Cryptic }_{\text {potential }} \\
\text { Thermophilic }\end{array}$ & & f.spp. & f.spp. & cl. sp. & ch.spp. & ch.spp. & $2 s p .^{\mathrm{b}}$ & b.spp. & $\begin{array}{l}\text { b.spp. } \\
\text { m.sp. }\end{array}$ \\
\hline \multicolumn{10}{|l|}{ Accumulation } \\
\hline $\mathrm{PHB}_{\max }$ & [wt \%] & $53 \pm 2$ & $75 \pm 2$ & $86 \pm 1$ & $91 \pm 1$ & $90 \pm 1$ & $90 \pm 1$ & $55 \pm 2$ & $46 \pm 3$ \\
\hline PHB:X & {$\left[g_{\mathrm{PHB}}: 1 \mathrm{gX}\right]$} & 1.1 & 3.1 & 6.1 & 10.5 & 9.4 & 9.4 & 1.2 & 0.9 \\
\hline
\end{tabular}

Acinetobacter (a.spp.), Zoogloea (z.sp.), Plasticicumulans acidivorans (p.a.), Flavobacterium (f.spp.), Cloacibacterium (cl.sp.), Chryseobacterium (ch.spp.), Bergeyella (b.spp.), Meiothermus (m.sp.)

${ }^{a}$ Dominant microorganisms as derived from relative abundance

${ }^{b}$ Hoarding: Plasticicumulans acidivorans and Nesiotobacter, potentially cryptic growth: Chryseobacterium and Alicycliphilus

\section{Overview of structural and functional development of the microbial communities}

An overview of the development of the respiration ratio $\left(\mathrm{OTR}_{\text {ratio }}\right)$ for all bioreactors is shown in Fig. 4. The respiration ratio correlates well with the observed functionality (distinguishing growing and hoarding strategies), thereby also serving as a process stability indicator. R25 and R30B show very comparable dynamics and community structure as is described for R30A, with a clear shift towards the hoarding strategy in P. acidivorans dominated systems that furthermore are functionally and microbially very stable systems (Pearson $r=0.908$ and $r=0.800$, respectively, with $p \ll 0.001$ and $n=18$ ). R35 has a slower transition towards the hoarding strategy. The gradual increase in the hoarding strategy aligns with the appearance of Nesiotobacter and Alicycliphilus (Pearson $r=0.637$ and $r=0.611$, respectively, with $p<0.01$ and $n=18$ ). The rapid shift towards the hoarding strategy after 120 cycles was unfortunately not covered by the $16 \mathrm{~S}$ amplicon sequencing.

The bioreactors operated at $40{ }^{\circ} \mathrm{C}$ show a stable and comparable community structure from cycle 10 onward. A gradual functional shift takes place over 150 cycles, in which the feast length decreases and the respiration ratio increases, but no change in the $\mathrm{O}_{2 \text {,feast }} \%$ is observed. Throughout the enrichment, no PHB is detected at the end of the feast phase in both bioreactors. Despite the absence of PHB, R40A consumes more oxygen in the famine phase (20-32\%) than a community with a typical growth strategy. Furthermore, a significant amount of ammonium is released (30-55\% of ammonia taken up in the feast phase) and the

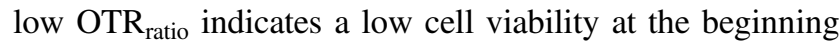
of the operational cycle. The data suggest abundance of a third competitive strategy characterized by an important role of cell decay and endogenous respiration.

Minor amounts of PHB were observed in the bioreactors operated at $20^{\circ} \mathrm{C}$. The increase in the $\mathrm{OTR}_{\text {ratio }}$ after 100 cycles indicates a shift towards the hoarding strategy for both bioreactors. From cycle 120 onwards, the two bioreactors diverge into different functional states; R20A transitions back to the growth strategy and R20B reaches a pseudo-steady state where the hoarding and growing strategy co-occur. The observed feast length for the cultures at $20^{\circ} \mathrm{C}$ is highly comparable. Community data 


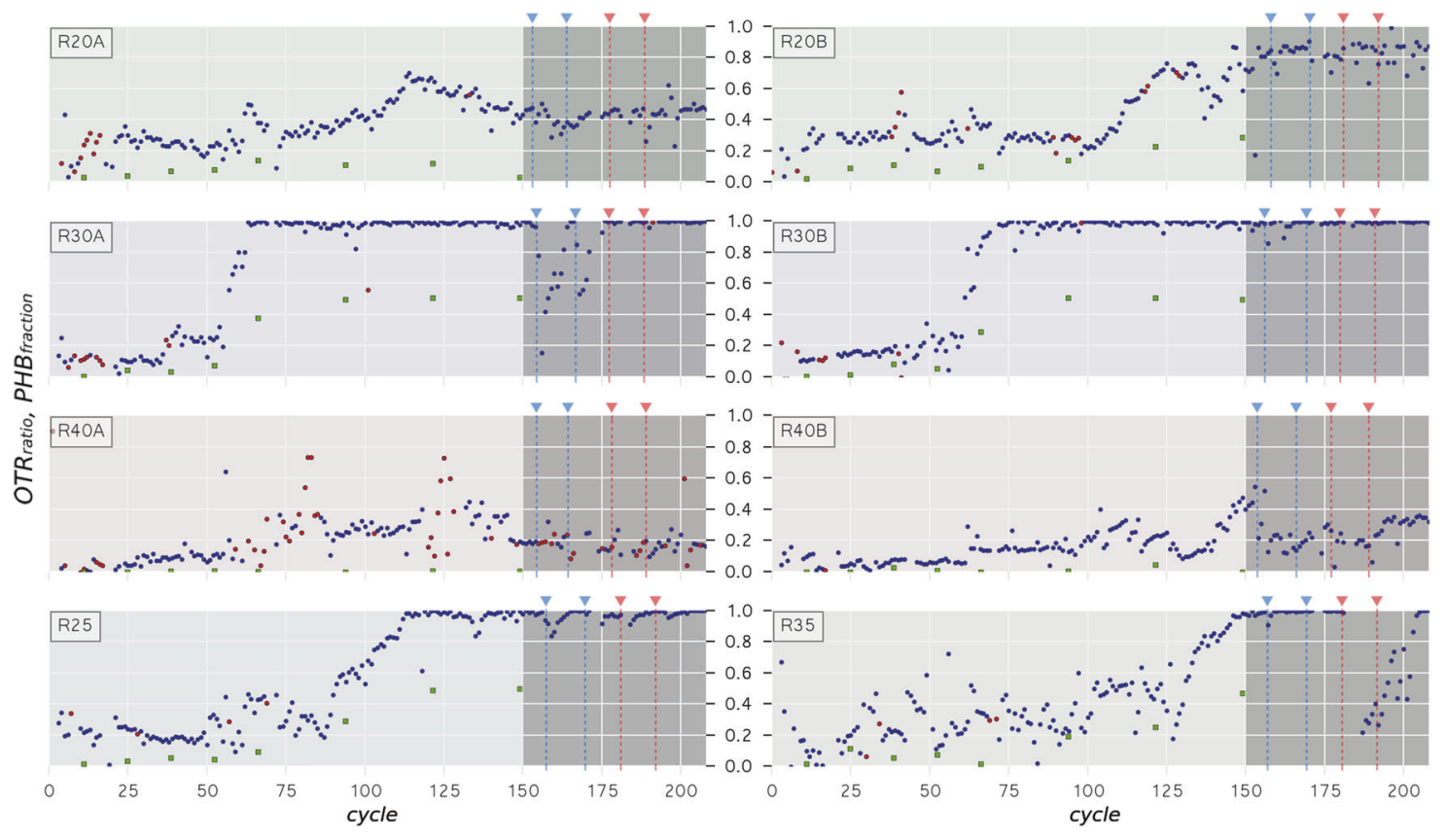

Fig. 4 Characteristic normalized change in oxygen transfer rate throughout the feast phase $\left(\mathrm{OTR}_{\text {ratio }}\right)$ for eight bioreactors over 150 cycles of enrichment (light) and 58 cycles of temperature disturbance

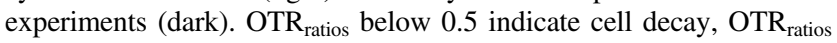

analyses (Supplementary Figs. S1-S2) suggest that both bioreactors demonstrate a strongly variable microbial community structure throughout the first 100 cycles. No clear enrichment of a limited number of species is observed, as opposed to most other operational temperatures. The observed functional shift after 100 cycles is associated with the appearance of Zoogloea in both bioreactors (Pearson $r=0.659$ and $r=0.816$, respectively, with $p<0.01$ and $p \ll 0.001$ and $n=18$ ). Whereas Zoogloea remained abundant in $\mathrm{R} 20 \mathrm{~B}$, a decrease in Zoogloea abundance in R20A after 20 cycles was observed and aligns with the drop in $\mathrm{OTR}_{\text {ratio. }}$. The eventual community composition of $\mathrm{R} 20 \mathrm{~B}$ seems stable and is composed of multiple members, resulting in a combined growing and hoarding functionality.

\section{Response to temperature shifts}

Short-term temperature shift experiments were carried out to investigate the temperature dependency of the processes observed. During the experiments, the temperature was either increased or decreased by $5{ }^{\circ} \mathrm{C}$ for two cycles, indicated with red and blue triangles in Fig. 4. Biomass in bioreactors R30A and R35 was strongly affected by the temperature disturbance during the second cycle, and required approximately ten cycles to recover. No change of functional strategy was observed during the first feast phase of the temperature shift experiments, but close to 1 indicate PHB production. The intermittently measured PHB fraction $\left(g_{\mathrm{PHB}} / g_{\mathrm{VSS}}\right)$ at the end of the feast phase is plotted as solid green squares

kinetic changes could readily be identified from online measurements.

The biomass specific substrate uptake rates $\left(q_{\mathrm{s}}\right)$ changed during the temperature shift experiments (Table 2). Generally, all biomass specific rates increased at higher temperatures and decreased at lower temperatures [38]. The only exception was the decrease (up to $40 \%$ ) in biomass specific conversion rates when the temperature of the bioreactors operated at 35 and $40^{\circ} \mathrm{C}$ was increased to 40 and $45^{\circ} \mathrm{C}$, respectively.

The biomass in $\mathrm{R} 25$, when shifted to $20^{\circ} \mathrm{C}$ achieved a biomass specific substrate uptake rate $80 \%$ higher than the highest rate observed with biomass enriched at $20^{\circ} \mathrm{C}$. The same occurred for the shifts from 30 to $35^{\circ} \mathrm{C}$, achieving a $70 \%$ higher biomass specific substrate uptake rate than the enrichment at $35^{\circ} \mathrm{C}$.

\section{Maximum PHB storage capacity}

To assess the maximum PHB production capacity of the cultures, ammonia-limited fed-batch experiments were performed at the end of the enrichment. The limitation of growth due to ammonia limitation constrains the metabolism of cells to nongrowth-related pathways, such as PHB production. All eight enrichments yielded biomass that produces the storage compound PHB (Table 1 and Supplementary Fig. S10). The four highest performing bioreactors, R25, R30A,B and R35, reached 86-91 $\pm 1 \mathrm{wt} \%$ 
Table 2 Short-term temperature change experiment. The middle column represents the biomass specific substrate uptake rate of the biomass culture that is obtained at the end of the enrichment (average of ten consecutive cycles, standard deviation in parenthesis). The left and right column represent the biomass specific uptake rates derived for the temperature shifts experiments $\left(-5\right.$ and $+5^{\circ} \mathrm{C}$ ) (average and standard deviation of two cycles). The cells in red did not follow the general Arrhenius relationship. Cells in green did, with the different shades representing how competitive the culture is with respect to long-term enrichments

\begin{tabular}{ccccc}
$\mathrm{T}$ & -5 & $\mathrm{~T}_{0}$ & +5 \\
\hline 20 & $0.4(0.2)$ & $0.7(0.2)$ & $1.2(0.5)$ \\
20 & $0.5(0.2)$ & $0.8(0.2)$ & $1.5(0.6)$ \\
\hline 25 & $1.5(0.2)$ & $1.9(0.4)$ & $2.2(0.2)$ \\
30 & $1.5(0.1)$ & $2.3(0.2)$ & $4.9(0.2)$ \\
30 & $1.7(0.1)$ & $2.3(0.2)$ & $4.8(0.2)$ \\
35 & $2.2(0.1)$ & $2.8(0.2)$ & $2.6(0.1)$ \\
40 & $2.7(0.1)$ & $3.5(0.3)$ & $2.0(0.1)$ \\
\hline 40 & $1.4(0.1)$ & $1.9(0.2)$ & $1.1(0.1)$
\end{tabular}

$\left(g_{\mathrm{PHB}} / g_{\mathrm{VSS}}\right)$. This is in the range of previously highest reported PHB storage capacities [14, 39]. The three lowest performing bioreactors, R20A, R40A, and R40B reached $46-53 \pm 2 \mathrm{wt} \%$. For reference: a $90 \%$ PHB accumulation is nine times more PHB per cell than a 50\% PHB accumulation (PHB:X $=9: 1$ vs 1:1).

The two biological replicates operated at $20{ }^{\circ} \mathrm{C}, \mathrm{R} 20 \mathrm{~A}$ and R20B, reach a PHB content of $53 \pm 2 \mathrm{wt} \%$ and $75 \pm 1$ wt\%, respectively. R20B produces three times as much PHB as its biological replicate, while the replicates at 30 and $40{ }^{\circ} \mathrm{C}$ do not differ significantly, and show very comparable production capacities.

\section{Discussion}

\section{Culture enrichments and bioreactor operation}

All bioreactors established a feast-famine regime within two operational cycles. As is shown in the overview plots, the competition for the limiting carbon substrate leads to a decreasing feast length demonstrating the enrichment of a faster community (Fig. 3 for R30A and Supplementary Figs. S1-S8 for other systems). There are two strategies for effective competition for acetate: the growing strategy and the hoarding strategy. Two online data derived functional indicators-OTR ratio and $\mathrm{O}_{2 \text {,feast }} \%$-allow for a direct interpretation whether PHB is produced as intermediate storage polymer, and differentiation between the growing and hoarding strategy. In time, microbial communities grown at temperatures from 25 to $35^{\circ} \mathrm{C}$ changed towards the hoarding strategy (Table 1). In general, higher biomass specific rates and decreased overall biomass yield values were identified at increasing temperatures, in line with the generalizations proposed by Heijnen and Kleerebezem [40]. The temperature shift experiments resulted in a typical increase and decrease of the biomass specific rates, except above $35^{\circ} \mathrm{C}$, where a temperature increase of $5{ }^{\circ} \mathrm{C}$ resulted in decreased biomass specific rates (Table 2). In addition, the hoarding biomass enriched at $25^{\circ} \mathrm{C}$, when incubated to $20^{\circ} \mathrm{C}$, had a biomass specific substrate uptake rate two times higher than biomass enriched at $20^{\circ} \mathrm{C}$. This suggests that the reactors operated at $20^{\circ} \mathrm{C}$ could also have enriched the faster hoarding microbial community as obtained at $25^{\circ} \mathrm{C}$. This observation, and the surprising absence of the hoarding strategy at $40{ }^{\circ} \mathrm{C}$, and only intermittently at $20^{\circ} \mathrm{C}$ form the basis of further discussion.

\section{Hoarding versus growth as dominant competitive strategy}

Enrichments with a short biomass retention time, based on one carbon source and electron donor, such as acetate, and oxygen as electron acceptor are highly selective and therefore one may expect a low biological diversity if one strain is outcompeting other microorganisms. Furthermore, the feast-famine strategy enriches microorganisms with the highest overall substrate uptake rate, which is directly reflected in the length of the feast phase. From a reductionist point of view, we may argue that producing catalytic biomass from acetate involves more complex metabolic machinery and therefore has a larger chance of being rate limiting than the relative short pathway from acetate to the storage polymer PHB. Theoretically this results in a trade-off between a high biomass specific substrate uptake rate $\left(q_{\mathrm{s}}\right)$ with PHB production (hoarding strategy) versus a lower $q_{\mathrm{s}}$ with an increasing number of catalysts (growing strategy). As far as we can see there is no intrinsic reason why this trade-off would be temperature dependent. Though, for the inoculum that was used in this study, the hoarding strategy was only dominant in the temperature range of $25-35^{\circ} \mathrm{C}$. Surprisingly, no PHB hoarding species were able to dominate at 20 and $40{ }^{\circ} \mathrm{C}$, where the growth strategy prevailed. Although the enrichments were operated for more than 75 generations and the inoculum contained a highly diverse microbial community (see Supplementary Materials), it is possible that the observations are inoculum specific. Nevertheless, all enriched communities were able to produce PHB when exposed to ammonium limitation, demonstrating the widespread abundance of the PHB production and the 
degradation pathway in prokaryotes (Table 1 and Supplementary Fig. S10). Evidently, the dominance of the hoarding versus growing strategy results from different kinetic properties and/or different pathway regulation in the different communities enriched at different temperatures. In other words, the competitive strength of both strategies apparently is not homogeneously distributed in all temperature domains. This means that no single competitive strategy consistently leads to dominance in feast-famine systems.

\section{Functionality and abundance}

The high number of sequence reads was successfully used to analyze the dynamics within the microbial communities throughout the enrichments. Logarithmic presentation of the data allows a visual alignment of a change in time of functional properties and populations within the reactors (Fig. 3e and Supplementary Figs. S1-S8). The resulting data suggest that much of the microbial community dynamics cannot be explained only by differences in kinetic and stoichiometric parameters. The general absence of clear successions of dominant community members and the persistent co-occurrence of microbial species and their oscillating relative abundance, suggests that processes like mutualism, adaptation, decay, and predation may play a significant role in the enrichment process [41, 42].

Even though changes in time can effectively be analyzed with NGS based methods, a major limitation of the NGS methodology used in this study remains that no reliable quantitative distribution of OTU's in time is possible. Herewith we cannot distinguish between enrichment of one strain with satellite populations, versus two or more dominant strains, and the origin of functional interdependencies remain unclear.

The strong correlation of the online data with population shifts allows for better sampling and 16S rRNA gene analysis strategies in future experiments (see Supplementary Table 2 for Pearson correlations). For example, the functionality shift from cycle 120 to 150 in bioreactor R35 is currently not captured due to the predetermined $16 \mathrm{~S}$ sampling strategy. With the online parameter identification, it is possible to collect frequent samples and analyze them at a high resolution in order to capture the specific regions of interest where functional shifts are identified with online measurements.

With the current sampling strategy, the community dynamics that occur within one cycle can also not be distinguished. More complex microbial community dynamics could involve a combination of growth on acetate, hoarding, decay, and cryptic growth. Analyzing multiple samples throughout one cycle could help to distinguish and identify the activity and abundance of specific populations by their change in relative abundance throughout one cycle.

\section{Cryptic growth as a third competitive strategy}

Although the majority of the functional responses of the bioreactors can be attributed to the presence of growers and hoarders, there are three observations that suggest that cell viability is a key biological factor that complicates the interpretation of the results in terms of microbial competition in sequencing batch bioreactors. First, all bioreactors show a decreased respiration ratio $\left(\mathrm{OTR}_{\text {ratio }}\right.$ in Fig. 4) during the initial cycles, and the higher temperature bioreactors maintain these low respiration ratios throughout the cultivation period. OTR $\mathrm{Tatio}$ values lower than 0.5 indicate that biological activity more than doubles throughout the feast phase, while only half of the biomass is removed. This suggests that some form of biomass inactivation, decay and/ or endogenous respiration takes place during the famine phase, resulting in a lower respiration rate at the beginning of the cycle than expected based on exchange ratios.

Second, the ammonia that is released during the famine phases of bioreactors $\mathrm{R} 40 \mathrm{~A}$ and $\mathrm{R} 40 \mathrm{~B}$, and to a lesser extent in R35, is likely coming from respired proteins and cell material. The biomass in bioreactor R40A, for example, releases more than half of the ammonium consumed in the feast phase, which suggests a viability of less than $40 \%$ at the end of the cycle.

Third, the relatively high abundance of microbial species, under which those from the family of Flavobacteriaceae, that are associated with growth on macromolecules such as proteins and polysaccharides, suggest that cell lysis and cryptic growth occur in many bioreactor systems (see Supplementary Information) [43]. Further differentiation of the abundance and strategies of the observed microbial species and their role in the microbial community remains unclear and would require a combination of metagenomic and metatranscriptomic analyses or an analysis of the community development during the cycle.

From these observations, we hypothesize that a third competitive strategy emerges as a result of biomass decay, lysis, and growth on the cellular constituents released in the famine phase, i.e, cryptic growth. (Table 1). This could explain the persistent microbial diversity that is observed in very selective microbial ecosystems, as described in this work, and the relatively high respiration rate in the famine phase in R40A and R40B in the absence of PHB. The low $\mathrm{OTR}_{\text {ratio }}$ observed in the initial cycles of all systems suggests that cell viability and cryptic growth could play a significant role in the biodiversity of pulse-fed bioreactors, even at moderate temperatures. As mentioned before, future research could specifically be aimed to elucidate this behavior by, for example, utilizing whole genome 
sequencing techniques. In addition, the low cell viability of biomass at higher temperatures allows for a mechanistic explanation as to why hoarding is no preferred strategy at higher temperatures in pulse-fed systems. A further discussion on this third competitive strategy can found in the Supplementary Materials and Fig. S9.

\section{Reproducibility of enrichments}

The three pairs of biological replicates developed differently throughout the enrichment, but reached comparable functional outcomes after 150 cycles, with some notable differences, as will be discussed below. During the cycle measurements of R40A and R40B, two different growing functionalities are observed: the shorter feast length and lower cell viability of bioreactor R40A versus the longer feast length and higher cell viability of bioreactor R40B. Throughout the enrichments, both functionalities are observed in both systems at varying times resulting in a relatively stable length of the feast phase at variable OTRratio values. The 16S rRNA gene analysis indicates that both mesophilic and thermophilic bacteria are present in both bioreactors (Supplementary Figs. S5-S6), which may indicate that community members are competitive by either having a fast biomass specific uptake rate, or high cell viability.

The biological replicates at $30^{\circ} \mathrm{C}$ developed in a very similar fashion, both functionally as well as structurally. Both bioreactors shift at approximately the same time after inoculation and take approximately ten cycles to shift from growth to storage as main competitive strategy. The high synchronicity of these bioreactors is likely due to the parallel enrichment of $P$. acidivorans in both bioreactors, as its dominance is associated with a feast length reduction of $\sim 30 \%$. P. acidivorans is well-known for its superior PHBproducing capabilities resulting in a maximum PHB content of up to $90 \mathrm{wt} \%$ under nutrient (i.e., ammonium) limiting conditions (Table 1 and Supplementary Fig. S10) [44].

The enrichments performed at $20^{\circ} \mathrm{C}$ resulted in a mixed characteristic of growth and hoarding after 150 cycles of enrichment, with emphasis on growth for bioreactor R20A and hoarding for bioreactor R20B. Both bioreactors also show ongoing community dynamics throughout the experimental period, indicating highly competitive conditions. This is reflected in the highly comparable length of the feast phase of bioreactors R20A and R20B. Jiang et al. [14] managed to achieve a functionally stable enrichment at $20^{\circ} \mathrm{C}$ within 50 operational cycles. Their microbial community was dominated by Zoogloea, as visually verified with FISH, but no $16 \mathrm{~S}$ rRNA gene analysis is available to compare their findings with our community structure. Their setup was highly comparable with ours, but was augmented with enriched hoarding biomass, which may explain the longer period required for Zoogloea to become dominant in our bioreactor, but it does not explain why Zoogloea did not become dominant from cycle 100 onwards.

In general, we observe significant variations in bioreactor functionality and diversity in some temperature domains, while other temperatures yield highly reproducible results. This indicates that there are still many factors left ununderstood, already in these relatively simple systems. A major improvement with this rigorous approach is that we can pinpoint specific circumstances where our knowledge is lacking.

\section{Concluding remarks}

Parallel cultivation from the same starting point (inoculum) allows for effective investigation of the functional development of microbial communities grown in variable environmental conditions. By combining functional system analysis based on online data with frequent community structure analysis by $16 \mathrm{~S}$ rRNA gene amplicon sequencing, comparative system development upon variation of a single variable can be studied in an effective way. Here we investigated pulse-fed selective conditions at five different temperatures in eight bioreactors. Exploration of competitive strategies benefit from the rigorous approach described here and will greatly contribute to our understanding of microbial ecosystem development and functioning, and subsequent development of bioprocesses.

The competitive strategies identified in pulse-fed bioreactors can be divided in three types: (i) direct growth on the primary substrate, (ii) hoarding of primary substrate and subsequent growth on storage polymers, and (iii) growth on the primary substrate combined with subsequent decay and cryptic growth. These three functional properties can, to a large extent, be directly identified from online data.

All communities were able to produce PHB to at least 50\% dry weight when incubated in excess of carbon substrate and ammonium limitation demonstrating the ubiquity of bacterial PHB production. Still, the microbial communities established in some bioreactors did not show any PHB production during the enrichment and therefore their PHB production mechanism played no role in the competition. Communities that produced PHB during the cycles, were also able to reach the highest PHB content during the accumulation experiments. The utilization of the PHB machinery as functional strategy to outcompete other microorganisms appears to have a temperature optimum at $30{ }^{\circ} \mathrm{C}$ in pulse-fed bioreactors. Dominance of the hoarding strategy is correlated with a high relative abundance of $P$. acidivorans.

The different developments of biological replicates from the same inoculum indicate that stochastic processes dominate the initial enrichment period. Replicates generally achieve comparable functional and microbial characteristics. 


\section{Data availability}

Sequence data collected for this study have been deposited in GenBank with BioProject ID PRJNA448046 and SRA Study SRP137048. Datasets of online measurements of 1824 cycles $(8 \times 228)$ are available at https://doi.org/10.4121/uuid: 499ec85a-6d8c-4c02-85c1-f932df4452ef as HDF5 dataset. All other data generated or analyzed during this study are included in this published article and its supplementary materials files, available at the ISME Journal's website.

Acknowledgements We gratefully acknowledge financial support from Netherlands Organization for Scientific Research (NWO) which is funded by the Ministry of Economic Affairs, and the company Paques B.V. (Paques Partnership Program project 13002).

\section{Compliance with ethical standards}

Conflict of interest The authors declare that there is no conflict of interest regarding the publication of this article.

Publisher's note: Springer Nature remains neutral with regard to jurisdictional claims in published maps and institutional affiliations.

\section{References}

1. Torsvik V, Øvreås L, Thingstad TF. Prokaryotic diversity-magnitude, dynamics, and controlling factors. Science. 2002;296:1064-6.

2. Cordero OX, Polz MF. Explaining microbial genomic diversity in light of evolutionary ecology. Nat Rev Microbiol. 2014;12:263-73.

3. Mitri S, Richard Foster K. The genotypic view of social interactions in microbial communities. Annu Rev Genet. 2013;47:247-73.

4. Carballa M, Regueiro L, Lema JM. Microbial management of anaerobic digestion: exploiting the microbiome-functionality nexus. Curr Opin Biotechnol. 2015;33:103-11.

5. Briones A, Raskin L. Diversity and dynamics of microbial communities in engineered environments and their implications for process stability. Curr Opin Biotechnol. 2003;14:270-6.

6. Perez-Garcia O, Lear G, Singhal N. Metabolic network modeling of microbial interactions in natural and engineered environmental systems. Front Microbiol. 2016;7:673.

7. Shin NR, Whon TW, Bae JW. Proteobacteria: microbial signature of dysbiosis in gut microbiota. Trends Biotechnol. 2015;33:496-503.

8. Bender SF, Wagg C, van der Heijden MGA. An underground revolution: biodiversity and soil ecological engineering for agricultural sustainability. Trends Ecol Evol. 2016;31:440-52.

9. Wagner M, Loy A, Nogueira R, Purkhold U, Lee N, Daims H. Microbial community composition and function in wastewater treatment plants. Antonie Van Leeuwenhoek. 2002;81:665-80.

10. Barr JJ, Slater FR, Fukushima T, Bond PL. Evidence for bacteriophage activity causing community and performance changes in a phosphorus-removal activated sludge. FEMS Microbiol Ecol. 2010;74:631-42.

11. Chen Y, Cheng JJ, Creamer KS. Inhibition of anaerobic digestion process: a review. Bioresour Technol. 2008;99:4044-64.

12. Stouten GR, Douwenga S, Hogendoorn C, Kleerebezem R. Dynamic biological system characterization through automated online data analysis. https://www.nature.com/ismej/authors-andreferees/gta, http://blogs.nature.com/nautilus/2007/01/citing submitted_but_not_publi.html.
13. Johnson K, Jiang Y, Kleerebezem R, Muyzer G, Van Loosdrecht MCM. Enrichment of a mixed bacterial culture with a high polyhydroxyalkanoate storage capacity. Biomacromolecules. 2009; 10:670-6.

14. Jiang Y, Marang L, Kleerebezem R, Muyzer G, Van Loosdrecht MCM. Effect of temperature and cycle length on microbial competition in PHB-producing sequencing batch reactor. ISME J. 2011;5:896-907.

15. Jiang Y, Marang L, Tamis J, Van Loosdrecht MCM, Dijkman H, Kleerebezem R. Waste to resource: converting paper mill wastewater to bioplastic. Water Res. 2012;46:5517-30.

16. Tamis J, Lužkov K, Jiang Y, Loosdrecht MCMva, Kleerebezem R. Enrichment of Plasticicumulans acidivorans at pilot-scale for PHA production on industrial wastewater. J Biotechnol. 2014;192:161-9.

17. Kleerebezem R, van Loosdrecht MC. Mixed culture biotechnology for bioenergy production. Curr Opin Biotechnol. 2007;18: 207-12.

18. Chen G-Q. A microbial polyhydroxyalkanoates (PHA) based bioand materials industry. Chem Soc Rev. 2009;38:2434.

19. Vishniac W, Santer M. The Thiobacilli. Microbiol Mol Biol Rev. 1957;21:195-213.

20. Beun J, Dircks K, Van Loosdrecht MCM, Heijnen J. Poly- $\beta$ hydroxybutyrate metabolism in dynamically fed mixed microbial cultures. Water Res. 2002;36:1167-80.

21. Johnson K, Kleerebezem R, Van Loosdrecht MCM. Model-based data evaluation of polyhydroxybutyrate producing mixed microbial cultures in aerobic sequencing batch and fed-batch reactors. Biotechnol Bioeng. 2009;104:50-67.

22. Marang L, Van Loosdrecht MCM, Kleerebezem R. Combining the enrichment and accumulation step in non-axenic PHA production: cultivation of Plasticicumulans acidivorans at high volume exchange ratios. J Biotechnol. 2016;231:260-7.

23. Kuhn HJ, Cometta S, Fiechter A. Effects of growth temperature on maximal specific growth rate, yield, maintenance, and death rate in glucose-limited continuous culture of the thermophilic Bacillus caldotenax. Eur J Appl Microbiol Biotechnol. 1980;10:303-15.

24. Verheijen PJT. Data reconciliation and error detection. In: Metabolic Pathway Engineering Handbook. Smolke, C.D. (ed.). Boca Raton: CRC Press; 8.1-8.13.

25. Caporaso JG, Lauber CL, Walters WA, Berg-Lyons D, Huntley J, Fierer N, et al. Ultra-high-throughput microbial community analysis on the Illumina HiSeq and MiSeq platforms. ISME J. 2012;6:1621-4.

26. Edgar RC. Search and clustering orders of magnitude faster than BLAST. Bioinformatics. 2010;26:2460-1.

27. Cole JR, Wang Q, Fish JA, Chai B, McGarrell DM, Sun Y, et al. Ribosomal database project: data and tools for high throughput rRNA analysis. Nucleic Acids Res. 2014;42:D633-42.

28. Caporaso JG, Kuczynski J, Stombaugh J, Bittinger K, Bushman FD, Costello EK, et al. QIIME allows analysis of high-throughput community sequencing data. Nat Methods. 2010;7:335-6.

29. Edgar RC. MUSCLE: multiple sequence alignment with high accuracy and high throughput. Nucleic Acids Res. 2004;32: 1792-7.

30. Brooks JP, Edwards DJ, Harwich MD, Rivera MC, Fettweis JM, Serrano MG, et al. The truth about metagenomics: quantifying and counteracting bias in $16 \mathrm{~S}$ rRNA studies. BMC Microbiol. 2015;15:66.

31. Sinha R, Abu-Ali G, Vogtmann E, Fodor AA, Ren B, Amir A, et al. Assessment of variation in microbial community amplicon sequencing by the Microbiome Quality Control (MBQC) project consortium. Nat Biotechnol. 2017;35:1077-86.

32. McBride MJ. The family flavobacteriaceae. In: The prokaryotes. Berlin, Heidelberg: Springer Berlin Heidelberg; 2014. p. 643-76. 
33. Towner KJ, Bergogne-Bérézin E, Fewson CA. Acinetobacter: portrait of a genus. In: The biology of acinetobacter. Boston, MA, US: Springer US; 1991. p. 1-24.

34. Tamaoka J, Ha D-M, Komagata K. Reclassification of Pseudomonas acidovorans den Dooren de Jong 1926 and Pseudomonas testosteroni Marcus and Talalay 1956 as Comamonas acidovorans comb. nov. and Comamonas testosteroni comb. nov. with an Emended Description of the Genus Comamonas. Int J Syst Bacteriol. 2009;37:52-59.

35. Kampfer P, Denner EBM, Meyer S, Moore ERB, Busse H-J. Classification of 'Pseudomonas azotocolligans' Anderson 1955, 132, in the genus Sphingomonas as Sphingomonas trueperi sp. nov. Int J Syst Bacteriol. 2009;47:577-83.

36. Jiang Y, Dimitry Y, Kleerebezem R, Muyzer G, van Loosdrecht M. Plasticicumulans acidivorans gen. nov., sp. nov., a polyhydroxyalkanoate-accumulating gammaproteobacterium from a sequencing-batch bioreactor. Int $\mathrm{J}$ Syst Evol Microbiol. 2011;61:2314-9.

37. Shin YK, Hiraishi A, Sugiyama J. Molecular systematics of the genus Zoogloea and emendation of the genus. Int J Syst Bacteriol. 2009;43:826-31.
38. Arrhenius S. Über die Reaktionsgeschwindigkeit bei der Inversion von Rohrzucker durch Säuren. Zeitschrift für Phys Chemie 1889;4: p. 226-48.

39. Johnson K, Kleerebezem R, Van Loosdrecht MCM. Influence of the $\mathrm{C} / \mathrm{N}$ ratio on the performance of polyhydroxybutyrate $(\mathrm{PHB})$ producing sequencing batch reactors at short SRTs. Water Res. 2010;44:2141-52.

40. Heijnen JJ, Kleerebezem R. Bioenergetics of microbial growth. In: Encyclopedia of industrial biotechnology. (Flickinger, M.C. and Drew, S.D., eds), John Wiley \& Sons 2010. p. 267-291.

41. Chacón JM, Harcombe WR. Antimicrobials: constraints on microbial warfare. Nat Microbiol. 2016;1:16225.

42. Baba T, Schneewind O. Instruments of microbial warfare: bacteriocin synthesis, toxicity and immunity. Trends Microbiol. 1998;6:66-71.

43. Aertsen A, Michiels CW. Stress and how bacteria cope with death and survival. Crit Rev Microbiol 2004;30:263-73.

44. Jiang Y, Marang L, Kleerebezem R, Muyzer G, van Loosdrecht MCM. Polyhydroxybutyrate production from lactate using a mixed microbial culture. Biotechnol Bioeng. 2011;108: 2022-35. 